\title{
A System of Generalized Variational-Hemivariational Inequalities with Set-Valued Mappings
}

\author{
Zhi-bin Liu, ${ }^{1}$ Jian-hong Gou, ${ }^{2}$ Yi-bin Xiao, ${ }^{3}$ and Xue-song $\mathrm{Li}^{4}$ \\ ${ }^{1}$ Southwest Petroleum University, State Key Laboratory of Oil and Gas Reservoir Geology and Exploitation, Chengdu, \\ Sichuan 610500, China \\ ${ }^{2}$ Southwest Petroleum University, The First National Gas Plant of ChangQing Oil Field Constituent Company, Xian, \\ Shanxi 750001, China \\ ${ }^{3}$ School of Mathematical Sciences, University of Electronic Science and Technology of China, Chengdu, Sichuan 611731, China \\ ${ }^{4}$ Department of Mathematics, Sichuan University, Chengdu, Sichuan 610064, China
}

Correspondence should be addressed to Yi-bin Xiao; xiaoyb9999@hotmail.com

Received 10 July 2013; Accepted 23 August 2013

Academic Editor: Shih-sen Chang

Copyright (C) 2013 Zhi-bin Liu et al. This is an open access article distributed under the Creative Commons Attribution License, which permits unrestricted use, distribution, and reproduction in any medium, provided the original work is properly cited.

By using surjectivity theorem of pseudomonotone and coercive operators rather than the KKM theorem and fixed point theorem used in recent literatures, we obtain some conditions under which a system of generalized variational-hemivariational inequalities concerning set-valued mappings, which includes as special cases many problems of hemivariational inequalities studied in recent literatures, is solvable. As an application, we prove an existence theorem of solutions for a system of generalized variationalhemivariational inequalities involving integrals of Clarke's generalized directional derivatives.

\section{Introduction}

Let $V_{1}, V_{2}, \ldots, V_{n}$ be real, separable, reflexive Banach spaces with dual spaces $V_{1}^{*}, V_{2}^{*}, \ldots, V_{n}^{*}$, and let $X_{1}, X_{2}, \ldots, X_{n}$ be real reflexive Banach spaces with dual spaces $X_{1}^{*}, X_{2}^{*}, \ldots, X_{n}^{*}$ such that there exist linear continuous and compact operators $T_{i}: V_{i} \rightarrow X_{i}$ for $i=1,2, \ldots, n$. We denote by $\langle\cdot, \cdot\rangle_{E^{*} \times E}$ the duality pairing between Banach space $E$ and its dual $E^{*}$ and by $\|\cdot\|_{E},\|\cdot\|_{E^{*}}$ the norms on the space $E$ and its dual space $E^{*}$, respectively, where $E=\left\{V_{i}, X_{i}, i=1,2, \ldots, n\right\}$. Assume that $A_{i}: \prod_{k=1}^{n} V_{k} \rightarrow 2^{V_{i}^{*}}, i=1,2, \ldots, n$ are set-valued mappings on the product space $\prod_{k=1}^{n} V_{k}$ of Banach spaces $V_{1}, \ldots, V_{n}$ and that $J: \prod_{k=1}^{n} X_{k} \rightarrow R$ is a functional on the product space $\prod_{k=1}^{n} X_{k}$ of Banach spaces $X_{1}, \ldots, X_{n}$, which is locally Lipschitz with respect to each component; that is, for all $i=1,2, \ldots, n$, the functionals $J\left(x_{1}, x_{2}, \ldots, x_{i-1}, \cdot, x_{i+1}, \ldots, x_{n}\right): X_{i} \rightarrow R$ are locally Lipschitz for all fixed $x_{j}, j \neq i$, and $G_{i}: V_{i} \rightarrow R \cup\{+\infty\}$, $i=1,2, \ldots, n$ are proper, convex, and lower semicontinuous functionals. In this paper, we study a system of generalized variational-hemivariational inequalities concerning setvalued mappings, which is specified as follows.

For all $i=1,2, \ldots, n$, find $u_{i} \in V_{i}$ and $\mu_{i} \in A_{i}(\mathbf{u})$ such that

(P)

$$
\begin{gathered}
\left\langle\mu_{i}, v_{i}-u_{i}\right\rangle_{V_{i}^{*} \times V_{i}}+J_{i}^{\circ}\left(\widehat{\mathbf{u}} ; \widehat{v}_{i}-\widehat{u}_{i}\right)+G_{i}\left(v_{i}\right) \\
-G_{i}\left(u_{i}\right) \geq 0, \quad \forall v_{i} \in V_{i},
\end{gathered}
$$

where $\mathbf{u}=\left(u_{1}, u_{2}, \ldots, u_{n}\right) \in \prod_{k=1}^{n} V_{k}, \widehat{\mathbf{u}}=\left(\widehat{u}_{1}, \widehat{u}_{2}, \ldots, \widehat{u}_{n}\right)=$ $\left(T_{1} u_{1}, T_{2} u_{2}, \ldots, T_{n} u_{n}\right), \widehat{v}_{i}=T_{i} v_{i}$ and $J_{i}^{\circ}\left(\widehat{\mathbf{u}} ; \widehat{v}_{i}-\widehat{u}_{i}\right)$ is the partial generalized directional derivative (in the sense of Clarke) of the functional $J$, which is locally Lipschitz for each component, with respect to the $i$ th component at the point $\widehat{u}_{i} \in X_{i}$ in the direction $\widehat{v}_{i}-\widehat{u}_{i}$ for all given $\widehat{u}_{j} \in X_{j}, j \neq i$, which can be defined by

$$
\begin{aligned}
J_{i}^{\circ}\left(\widehat{\mathbf{u}} ; \widehat{v}_{i}-\widehat{u}_{i}\right) \\
\quad=\limsup _{x \rightarrow \widehat{u}_{i}, \lambda \downarrow 0} \frac{J\left(\widehat{u}_{1}, \ldots, \widehat{u}_{i-1}, x+\lambda\left(\widehat{v}_{i}-\widehat{u}_{i}\right), \widehat{u}_{i+1}, \ldots, \widehat{u}_{n}\right)}{\lambda}
\end{aligned}
$$




$$
-\frac{J\left(\widehat{u}_{1}, \ldots, \widehat{u}_{i-1}, x, \widehat{u}_{i+1}, \ldots, \widehat{u}_{n}\right)}{\lambda} .
$$

In the last few years, there are many researchers who dedicated themselves to the study of various types of hemivariational inequalities and systems of hemivariational inequalities, which are a generalization of the variational inequalities, and related problems such as equilibrium problems. In these papers, based on Clarke's generalized directional derivative and Clarke's generalized gradient for locally Lipchitz functions, the researchers study the existence and uniqueness of solution by mainly using KKM theorems, surjectivity theorems for pseudomonotone and coercive operators, fixed point theorems, critical point theory, and so on. We refer readers for the study of hemivariational inequalities to monographs of Carl et al. [1], Migórski et al. [2], Naniewicz and Panagiotopoulos [3], and Panagiotopoulos [4]. For the system of hemivariational inequalities, Denkowski and Migórski [5] studied a dynamic thermoviscoelastic frictional contact problem which was modeled by a system of evolution hemivariational inequalities. They proved the existence and uniqueness of the weak solution for the problem by using a surjectivity result for operators of pseudomonotone type. In 2011, Repovš and Varga [6] studied the Nash equilibrium point by using the Ky Fan version of the KKM theorem and the Tarafdar fixed point theorem for a class of hemivariational inequality system. It is obvious that some problems studied in literatures are special cases of our system of generalized variational-hemivariational inequalities under some special conditions, such as $n=1, A_{i}$ are single-valued, or $G_{i}$ are indicators of some convex subsets $K_{i}$ for $i=1,2, \ldots, n$. Although it seems that our problem (P) cannot include the problem studied in [6] as a special case, we remark here that, in essence, the problem $(\mathrm{P})$ is a generalization of the problem in [6] since, when $A_{i}(i=1,2, \ldots, n)$ are single-valued and $G_{i}$ are the indicators of the convex subsets $K_{i}$, the problem (P) reduces to the problem studied by Repovš and Varga [6] with $T_{i}=S_{i}$ and $A_{i}^{\circ}\left(T u ; T_{i} v_{i}-T_{i} u_{i}\right)$ being incorporated into $J_{i}^{\circ}\left(S u ; S_{i} v_{i}-S_{i} u_{i}\right)$ under the regularity condition. For more information on the research of hemivariational inequalities and systems of hemivariational inequalities, we can refer to [7-16] and references therein.

It is well known that, by surjectivity theorem of pseudomonotone and coercive operators, there exists solution $u_{i}$ to each variational-hemivariational inequality in the system (1) for all $u_{j} \in V_{j}, j \neq i$ under some suitable conditions on the operators $A_{i}, J$, and $G_{i}$. A natural question is whether these conditions are sufficient for the existence of solutions to the system (1) which is combined by solvable variational-hemivariational inequalities. If not, what other stronger conditions do we need to guarantee the solvability of the system (1)? In this paper, we are devoted to these questions by using surjectivity theorem of pseudomonotone and coercive operators rather than the KKM theorem, and the fixed point theorem used by Repovš and Varga in [6] to obtain the existence of the solutions to the problem (P) of a system of generalized variational-hemivariational inequalities concerning set-valued mappings.
As will be seen in the proof of our main theorem in Section 3, the case where $n=k$ for any finite positive integer $k>2$ is a natural generalization of the case where $n=2$. Therefore, in what follows, We will focus on the problem of a system of two generalized variational-hemivariational inequalities, which can be reformulated as follows. Consider $u_{1} \in V_{1}, u_{2} \in V_{2}, \mu_{1} \in A_{1}(u)$ and $\mu_{2} \in A_{2}(u)$ such that

$$
\left(\mathrm{P}^{\prime}\right)\left\{\begin{array}{cc}
\left\langle\mu_{1}, v_{1}-u_{1}\right\rangle_{V_{1}^{*} \times V_{1}}+J_{1}^{\circ}\left(\widehat{u} ; \widehat{v}_{1}-\widehat{u}_{1}\right) & \\
+G_{1}\left(v_{1}\right)-G_{1}\left(u_{1}\right) \geq 0, & \forall v_{1} \in V_{1}, \\
\left\langle\mu_{2}, v_{2}-u_{2}\right\rangle_{V_{2}^{*} \times V_{2}}+J_{2}^{\circ}\left(\widehat{u} ; \widehat{v}_{2}-\widehat{u}_{2}\right) & \\
+G_{2}\left(v_{2}\right)-G_{2}\left(u_{2}\right) \geq 0, & \forall v_{2} \in V_{2},
\end{array}\right.
$$

where $u=\left(u_{1}, u_{2}\right), \widehat{u}=\left(\widehat{u}_{1}, \widehat{u}_{2}\right)=\left(T_{1} u_{1}, T_{2} u_{2}\right)$ and $\widehat{v}_{i}=T_{i} v_{i}$ for $i=1,2$.

The paper is structured as follows. In Section 2, we recall some preliminary material. Section 3 gives conditions under which the problem (P) of a system of generalized variationalhemivariational inequalities concerning set-valued mapping is solvable by considering the simple case, the problem $\left(\mathrm{P}^{\prime}\right)$ of a system of two generalized variational-hemivariational inequalities. At last, in Section 4, we are concerned with an application of our results to a system of generalized variational-hemivariational inequalities involving integrals of Clarke's generalized directional derivatives.

\section{Preliminaries}

In this section, we recall some important notations and useful results on nonlinear analysis, nonsmooth analysis, and operators of monotone type, which can be found in $[2,3,17$, $18]$.

Without confusion of symbols, we suppose, just in this section, that $X$ is a Banach space with its dual $X^{*}$ and duality paring $\langle\cdot, \cdot\rangle$ between $X^{*}$ and $X, G: X \rightarrow R \cup\{+\infty\}$ is a proper and convex functional, and $J: X \rightarrow R$ is a locally Lipschitz functional with Clarke's generalized directional derivative $J^{\circ}(u, v)$. We denote by $\widehat{\partial} G(u): X \rightarrow$ $2^{X^{*}} \backslash\{\emptyset\}$ and $\partial J(u): X \rightarrow 2^{X^{*}} \backslash\{\emptyset\}$ the subgradient of the convex functional $G$ in the sense of convex analysis and Clarke's generalized gradient of the locally Lipschitz functional $J$, respectively. Then,

$$
\begin{gathered}
\widehat{\partial} G(u)=\left\{u^{*} \in X^{*}: G(v)-G(u)\right. \\
\left.\geq\left\langle u^{*}, v-u\right\rangle, \forall v \in X\right\}, \\
\partial J(u)=\left\{\omega \in X^{*}: J^{\circ}(u, v) \geq\langle\omega, v\rangle, \forall v \in X\right\} .
\end{gathered}
$$

We have the following basic properties on Clarke's generalized directional derivative and Clarke's generalized gradient (see, e.g., $[2,17]$ ).

Proposition 1. Let $X$ be Banach space, and let $u, v \in X$, and $J$ be locally Lipschitz functional defined on $X$. Then one has the following.

(1) The function $v \mapsto J^{\circ}(u, v)$ is finite, positively homogeneous, subadditive, and then convex on $X$; 
(2) $J^{\circ}(u, v)$ is upper semicontinuous as a function of $(u, v)$, but as a function of $v$ alone, it is Lipschitz continuous on $X$.

(3) $\partial J(u)$ is a nonempty, convex, bounded, and weak ${ }^{*}$ compact subset of $X^{*}$.

(4) For every $v \in X$, one has

$$
J^{\circ}(u, v)=\max \{\langle\xi, v\rangle: \xi \in \partial J(u)\} .
$$

(5) The graph of the Clarke generalized gradient $\partial J(u)$ is closed in $X \times\left(w^{*}-X^{*}\right)$ topology.

(6) The multifunction $X \ni u \rightarrow \partial J(u) \subseteq X^{*}$ is upper semicontinuous from $X$ into $w^{*}-X^{*}$.

Definition 2. A locally Lipschitz functional $J: X \rightarrow R$ is said to be regular (in the sense of Clarke) at $u \in X$ if

(i) for all $v \in X$ the directional derivative $J^{\prime}(u, v)$ exists;

(ii) for all $v \in X, J^{\prime}(u, v)=J^{\circ}(u, v)$,

where $J^{\prime}(u, v)$ is directional derivative of $J$ at $u \in X$ in the direction $v \in X$, which is defined by

$$
J^{\prime}(u, v)=\lim _{\lambda \downarrow 0} \frac{J(u+\lambda v)-J(u)}{\lambda},
$$

whenever this limit exists. The functional $J$ is regular (in the sense of Clarke) on $X$ if it is regular at every point $u \in X$.

Proposition 3. Let $X_{1}$ and $X_{2}$ be two Banach spaces. If $J$ : $X_{1} \times X_{2} \rightarrow R$ is locally Lipschitz and either $J$ or $-J$ is regular at $u=\left(u_{1}, u_{2}\right) \in X_{1} \times X_{2}$, then

$$
\partial J\left(u_{1}, u_{2}\right) \subseteq \partial_{1} J\left(u_{1}, u_{2}\right) \times \partial_{2} J\left(u_{1}, u_{2}\right),
$$

or equivalently one has

$$
\begin{array}{r}
J^{\circ}\left(u_{1}, u_{2} ; v_{1}, v_{2}\right) \leq J_{1}^{\circ}\left(u_{1}, u_{2} ; v_{1}\right)+J_{2}^{\circ}\left(u_{1}, u_{2} ; v_{2}\right), \\
\forall\left(v_{1}, v_{2}\right) \in X_{1} \times X_{2},
\end{array}
$$

where $\partial_{1}\left(u_{1}, u_{2}\right)$ (resp., $\left.\partial_{2}\left(u_{1}, u_{2}\right)\right)$ represents the partial generalized subgradient of $J\left(\cdot, u_{2}\right)$ (resp., $\left.J\left(u_{1}, \cdot\right)\right)$ and $J_{1}^{\circ}\left(u_{1}, u_{2} ; v_{1}\right)$ (resp., $\left.J_{2}^{\circ}\left(u_{1}, u_{2} ; v_{2}\right)\right)$ denotes the partial generalized directional derivative of $J\left(\cdot, u_{2}\right)$ (resp., $\left.J\left(u_{1}, \cdot\right)\right)$ at the point $u_{1}$ (resp., $\left.u_{2}\right)$ in the direction $v_{1}$ (resp., $\left.v_{2}\right)$, but the converse of inclusion (7) and inequality (8) is not true in general.

Definition 4. Let $X$ be real reflexive Banach space with dual $X^{*}$. A mapping $T$ from $X$ into $2^{X^{*}}$ is said to be pseudomonotone if

(1) the set $T u$ is nonempty, bounded, closed, and convex for all $u \in X$;

(2) $T$ is upper semicontinuous from each finite dimensional subspace of $X$ to $X^{*}$ endowed with the weak topology;
(3) $\left\{u_{i}\right\}$ is a sequence in $X$ converging weakly to $u$, and $u_{i}^{*} \in T u_{i}$ is such that

$$
\lim \sup \left\langle u_{i}^{*}, u_{i}-u\right\rangle \leq 0,
$$

then for each element $v \in X$ there exists $u^{*}(v) \in T u$ such that

$$
\liminf \left\langle u_{i}^{*}, u_{i}-v\right\rangle \geq\left\langle u^{*}(v), u-v\right\rangle .
$$

Definition 5. Let $X$ be real reflexive Banach space with dual $X^{*}$. A mapping $T$ from $X$ into $2^{X^{*}}$ is said to be generalized pseudomonotone if for any sequences $\left\{u_{i}\right\} \subset X,\left\{u_{i}^{*}\right\} \subset X^{*}$ with $u_{i}^{*} \in T u_{i}, u_{i} \rightarrow u$ weakly in $X, u_{i}^{*} \rightarrow u^{*}$ weakly in $X^{*}$ and

$$
\lim \sup \left\langle u_{i}^{*}, u_{i}-u\right\rangle \leq 0
$$

then one has $u^{*} \in T u$ and $\left\langle u_{i}^{*}, u_{i}\right\rangle \rightarrow\left\langle u^{*}, u\right\rangle$.

Proposition 6. Let $X$ be real reflexive Banach space with dual $X^{*}$ and let $T_{1}, T_{2}$ be two pseudomonotone mappings from $X$ into $2^{X^{*}}$. Then $T_{1}+T_{2}$ is pseudomonotone.

Proposition 7. Let $X$ be real reflexive Banach space with dual $X^{*}$ and let $T: X \rightarrow 2^{X^{*}}$ be a pseudomonotone mapping from $X$ into $2^{X^{*}}$. Then $T$ is a generalized pseudomonotone.

Proposition 8. Let $X$ be real reflexive Banach space with dual $X^{*}$ and let $T$ be a bounded, generalized pseudomonotone mapping from $X$ into $2^{X^{*}}$. Assume that, for each $u \in X$, $T u$ is a nonempty closed convex subset of $X^{*}$. Then $T$ is pseudomonotone.

Definition 9. Let $X$ be real reflexive Banach space with dual $X^{*}$. The operator $T: X \rightarrow 2^{X^{*}}$ is said to be as follows:

(1) monotone if for all $\left(u, u^{*}\right),\left(v, v^{*}\right)$ lying in the graph $G(T)$ of $T$, one has

$$
\left\langle u^{*}-v^{*}, u-v\right\rangle \geq 0 .
$$

(2) maximal monotone if it is monotone and if $\left(u, u^{*}\right) \epsilon$ $X \times X^{*}$ is such that

$$
\left\langle u^{*}-v^{*}, u-v\right\rangle \geq 0, \quad \forall\left(v, v^{*}\right) \in G(T) ;
$$

then $\left(u, u^{*}\right) \in G(T)$.

(3) quasibounded if for each $M>0$ there exists $K(M)>$ 0 such that, whenever $\left(u, u^{*}\right) \in G(T),\left\langle u^{*}, u\right\rangle \leq$ $M\|u\|$, and $\|u\| \leq M$; then

$$
\left\|u^{*}\right\| \leq K(M)
$$

(4) strongly quasibounded if for each $M>0$ there exists $K(M)>0$ such that for all $\left(u, u^{*}\right) \in G(T)$ with $\left\langle u^{*}, u\right\rangle \leq M$ and $\|u\| \leq M$, one has

$$
\left\|u^{*}\right\| \leq K(M) \text {. }
$$


Definition 10. Let $X$ be real reflexive Banach space with dual $X^{*}$. A mapping $T$ from $X$ into $2^{X^{*}}$ is said to be as follows:

(1) coercive if there exists a real-valued function $c$ on $R^{+}$ with $\lim _{r \rightarrow \infty} c(r)=\infty$ such that for all $\left(u, u^{*}\right) \in$ $G(T)$, one has

$$
\left\langle u^{*}, u\right\rangle \geq c(\|u\|)\|u\|
$$

(2) coercive with constant $\alpha>0$ if

$$
\left\langle u^{*}, u\right\rangle \geq \alpha\|u\|^{2}
$$

(3) $u_{0}$-coercive if there exists a real-valued function $c$ on $R^{+}$with $\lim _{r \rightarrow \infty} c(r)=\infty$ such that for some $u_{0} \in X$ and for all $\left(u, u^{*}\right) \in G(T)$, one has

$$
\left\langle u^{*}, u-u_{0}\right\rangle \geq c(\|u\|)\|u\| \text {. }
$$

The following theorem is a surjectivity theorem for the sum of a pseudomonotone, coercive operator, and a maximal monotone operator, which is important to the proof of our main results.

Theorem 11 (see [3]). Let $X$ be a real reflexive Banach space with dual $X^{*}$, let $\widehat{T}$ be a maximal monotone mapping from $X$ into $2^{X^{*}}$ with $u_{0} \in D(T)=\{x: T x \neq \emptyset\}$, and let $T$ be a $u_{0^{-}}$ coercive, pseudomonotone operator from $X$ into $2^{X^{*}}$. Suppose further that either $T_{u_{0}}: X \rightarrow 2^{X^{*}}$ is quasibounded or $\widehat{T}_{u_{0}}$ : $X \rightarrow 2^{X^{*}}$ is strongly quasibounded, where $T_{u_{0}}(v)=T\left(u_{0}+v\right)$ and the same for $\widehat{T}_{u_{0}}$. Then $R(T+\widehat{T})=X^{*}$.

\section{Main Results}

In this section, we first give an existence theorem for the solution to the problem $\left(\mathrm{P}^{\prime}\right)$ of a system of two generalized variational-hemivariational inequalities. And then, as a natural generalization, an existence theorem for the solution to the problem $(\mathrm{P})$, a system of generalized variationalhemivariational inequalities concerning set-valued mappings is also obtained.

Before we present the main existence theorem, for the simplicity of writing, we define some useful symbols and give a crucial lemma in advance, which establishes the relationship between the problem $\left(\mathrm{P}^{\prime}\right)$ of a system of two variational-hemivariational inequalities and a generalized vector variational-hemivariational inequality. Let $V=V_{1} \times V_{2}$. Endowed with the norm defined by

$$
\|u\|_{V}=\left\|u_{1}\right\|_{V_{1}}+\left\|u_{2}\right\|_{V_{2}}, \quad \forall u=\left(u_{1}, u_{2}\right) \in V
$$

$V$ is a reflexive Banach space with dual $V^{*}$. The duality pairing between $V$ and $V^{*}$ is given by

$$
\begin{array}{r}
\left\langle u^{*}, u\right\rangle_{V^{*} \times V}=\left\langle u_{1}^{*}, u_{1}\right\rangle_{V_{1}^{*} \times V_{1}}+\left\langle u_{2}^{*}, u_{2}\right\rangle_{V_{2}^{*} \times V_{2}}, \\
\forall u^{*}=\left(u_{1}^{*}, u_{2}^{*}\right) \in V^{*}, u=\left(u_{1}, u_{2}\right) \in V .
\end{array}
$$

On the Banach space $V$ defined above, we further define a set-valued mapping $A: V \rightarrow 2^{V^{*}}$, an operator $T: V \rightarrow$ $X_{1} \times X_{2}$, and a functional $G: V \rightarrow R \cup\{+\infty\}$, which are specified as follows. For all $u=\left(u_{1}, u_{2}\right) \in V$, one has

$$
\begin{aligned}
& A(u)=\left(A_{1}(u), A_{2}(u)\right), \\
& T(u)=\left(T_{1} u_{1}, T_{2} u_{2}\right), \\
& G(u)=G_{1}\left(u_{1}\right)+G_{2}\left(u_{2}\right) .
\end{aligned}
$$

Lemma 12. Assume that $G_{i}: V_{i} \rightarrow R \cup\{+\infty\}, i=1,2$ are proper, convex, and lower semicontinuous functionals. The functional $G$ defined above is also a proper, convex, and lower semicontinuous functional on $V$. Moreover, $\widehat{\partial} G(u)=\widehat{\partial} G_{1}\left(u_{1}\right) \times$ $\widehat{\partial} G_{2}\left(u_{2}\right)$.

Proof. Since the functionals $G_{i}: V_{i} \rightarrow R \cup\{+\infty\}, i=1,2$ are proper and convex, it is easy to show that $G$ is also proper and convex by the inequality $G(\lambda u+(1-\lambda v))=G_{1}\left(\lambda u_{1}+\right.$ $\left.(1-\lambda) v_{1}\right)+G_{2}\left(\lambda u_{2}+(1-\lambda) v_{2}\right) \leq \lambda G(u)+(1-\lambda) G(v)$ for all $u, v \in V$ and $\lambda \in[0,1]$. As for the lower semicontinuity of the functional $G$, by assuming that $u^{n} \rightarrow u$ in $V$, which implies $u_{1}^{n} \rightarrow u_{1}$ in $V_{1}$ and $u_{2}^{n} \rightarrow u_{2}$ in $V_{2}$, we can get from the lower semicontinuity of $G_{1}$ and $G_{2}$ that

$$
\begin{aligned}
\lim \inf G\left(u^{n}\right) & =\liminf \left(G_{1}\left(u_{1}^{n}\right)+G_{2}\left(u_{2}^{n}\right)\right) \\
& \geq \liminf G_{1}\left(u_{1}^{n}\right)+\liminf G_{2}\left(u_{2}^{n}\right) \\
& \geq G_{1}\left(u_{1}\right)+G_{2}\left(u_{2}\right)=G(u),
\end{aligned}
$$

which means that $G$ is lower semicontinuous on $V$.

Now, we prove the equality $\widehat{\partial} G(u)=\widehat{\partial} G_{1}\left(u_{1}\right) \times \widehat{\partial} G_{2}\left(u_{2}\right)$. Assume that $\mu \in \widehat{\partial} G(u) \subset V^{*}$, which says that

$$
G(v)-G(u) \geq\langle\mu, v-u\rangle_{V^{*} \times V}, \quad \forall v \in V .
$$

In particular, for any $v_{1} \in V_{1}$, let $v=\left(v_{1}, u_{2}\right)$ in (23), and then we can get that

$$
G_{1}\left(v_{1}\right)-G_{1}\left(u_{1}\right) \geq\left\langle\mu_{1}, v_{1}-u_{1}\right\rangle_{V_{1}^{*} \times V_{1}}, \quad \forall v_{1} \in V_{1} .
$$

Similarly, by letting $v=\left(u_{1}, v_{2}\right)$ in (23) for any $v_{2} \in V_{2}$, we can obtain

$$
G_{2}\left(v_{2}\right)-G_{2}\left(u_{2}\right) \geq\left\langle\mu_{2}, v_{2}-u_{2}\right\rangle_{V_{2}^{*} \times V_{2}}, \quad \forall v_{2} \in V_{2},
$$

which together with (24) implies that $\mu \in \widehat{\partial} G_{1}\left(u_{1}\right) \times \widehat{\partial} G_{2}\left(u_{2}\right)$; that is, $\widehat{\partial} G(u) \subseteq \widehat{\partial} G_{1}\left(u_{1}\right) \times \widehat{\partial} G_{2}\left(u_{2}\right)$.

Conversely, let $\mu=\left(\mu_{1}, \mu_{2}\right) \in \widehat{\partial} G_{1}\left(u_{1}\right) \times \widehat{\partial} G_{2}\left(u_{2}\right)$. For all $v_{i} \in V_{i}, i=1,2$, it follows from $\mu_{i} \in \widehat{\partial} G_{i}\left(u_{i}\right)$ that

$$
\begin{array}{ll}
G_{1}\left(v_{1}\right)-G_{1}\left(u_{1}\right) \geq\left\langle\mu_{1}, v_{1}-u_{1}\right\rangle_{V_{1}^{*} \times V_{1}}, & \forall v_{1} \in V_{1}, \\
G_{2}\left(v_{2}\right)-G_{2}\left(u_{2}\right) \geq\left\langle\mu_{2}, v_{2}-u_{2}\right\rangle_{V_{2}^{*} \times V_{2}}, & \forall v_{2} \in V_{2} .
\end{array}
$$

By adding the two inequalities (26), we obtain

$$
G(v)-G(u) \geq\langle\mu, v-u\rangle_{V^{*} \times V}, \quad \forall v \in V,
$$

which implies that $\mu \in \widehat{\partial} G(u)$; that is, $\widehat{\partial} G(u) \supseteq \widehat{\partial} G_{1}\left(u_{1}\right) \times$ $\widehat{\partial} G_{2}\left(u_{2}\right)$. This completes the proof of Lemma 12 . 
Now, we consider the following generalized vector variational-hemivariational inequality. Find $u=\left(u_{1}, u_{2}\right) \in V$ and $\mu=\left(\mu_{1}, \mu_{2}\right) \in A(u)$ such that

$$
\begin{array}{r}
\left(\mathrm{P}^{\prime \prime}\right) \quad\langle\mu, v-u\rangle_{V^{*} \times V}+J^{\circ}(T u, T v-T u)+G(v) \\
-G(u) \geq 0, \quad \forall v \in V .
\end{array}
$$

We first give a crucial lemma which establishes the relationship between the problem $\left(\mathrm{P}^{\prime}\right)$ of a system of two variational-hemivariational inequalities and the problem $\left(\mathrm{P}^{\prime \prime}\right)$ of a generalized vector variational-hemivariational inequality.

Lemma 13. Assume that the locally Lipschitz functional $J$ : $X_{1} \times X_{2} \rightarrow R$ is regular on $X_{1} \times X_{2}$. Then any solution $u=\left(u_{1}, u_{2}\right) \in V$ to the problem $\left(\mathrm{P}^{\prime \prime}\right)$ is always a solution to the problem $\left(\mathrm{P}^{\prime}\right)$.

Proof. Assume that $u=\left(u_{1}, u_{2}\right)$ solves the problem $\left(\mathrm{P}^{\prime \prime}\right)$, which says that there exists an $\mu=\left(\mu_{1}, \mu_{2}\right) \in A(u)$ such that for all $v \in V$, one has

$$
\langle\mu, v-u\rangle_{V^{*} \times V}+J^{\circ}(T u, T v-T u)+G(v)-G(u) \geq 0 .
$$

Specially, for any $v_{1} \in V_{1}$, let $v=\left(v_{1}, u_{2}\right) \in V$ in (29), and then we can get from Proposition 3 that

$$
\begin{aligned}
0 \leq & \left\langle\mu_{1}, v_{1}-u_{1}\right\rangle_{V_{1}^{*} \times V_{1}}+J^{\circ}\left(\mathrm{Tu},\left(T_{1} v_{1}-T_{1} u_{1}, 0\right)\right) \\
& +G_{1}\left(v_{1}\right)-G_{1}\left(u_{1}\right) \\
\leq & \left\langle\mu_{1}, v_{1}-u_{1}\right\rangle_{V_{1}^{*} \times V_{1}}+J_{1}^{\circ}\left(T u, T_{1} v_{1}-T_{1} u_{1}\right) \\
& +G_{1}\left(v_{1}\right)-G_{1}\left(u_{1}\right), \quad \forall v_{1} \in V_{1} .
\end{aligned}
$$

Similarly, by letting $v=\left(u_{1}, v_{2}\right) \in V$ in (29) for any $v_{2} \epsilon$ $V_{2}$, we can obtain that

$$
\begin{gathered}
\left\langle\mu_{2}, v_{2}-u_{2}\right\rangle_{V_{2}^{*} \times V_{2}}+J_{2}^{\circ}\left(T u, T_{2} v_{2}-T_{2} u_{2}\right) \\
+G_{2}\left(v_{2}\right)-G_{2}\left(u_{2}\right) \geq 0, \quad \forall v_{2} \in V_{2},
\end{gathered}
$$

which together with the inequality (30) implies that $u=$ $\left(u_{1}, u_{2}\right)$ is a solution to the problem $\left(\mathrm{P}^{\prime}\right)$. This completes the proof of Lemma 13.

Remark 14. It follows from Proposition 3 that, just under regularity condition of the functional $J, J^{\circ}(u, v)=J_{1}^{\circ}\left(u, v_{1}\right)+$ $J_{2}^{\circ}\left(u, v_{2}\right)$ does not hold in general, while the inequality $J^{\circ}(u, v) \leq J_{1}^{\circ}\left(u, v_{1}\right)+J_{2}^{\circ}\left(u, v_{2}\right)$ is true. Therefore, without other much stronger conditions on functional $J$, the inverse of the Lemma 13 is not true in general.

We give some assumptions on the operators $A_{i}$ and $J$ in the system (3) of two generalized variational-hemivariational inequalities.

The assumption (HA) is as follows.

(1) $A_{1}: V_{1} \times V_{2} \rightarrow 2^{V_{1}^{*}}$ is bounded on $V_{1} \times V_{2}$ and pseudomonotone with respect to the first argument; that is, for all $u_{2} \in V_{2}$, the operator $A_{1}\left(\cdot, u_{2}\right): V_{1} \rightarrow 2^{V_{1}^{*}}$ is pseudomonotone on $V_{1}$.

(2) $A_{2}: V_{1} \times V_{2} \rightarrow 2^{V_{2}^{*}}$ is bounded on $V_{1} \times V_{2}$ and pseudomonotone with respect to the second argument; that is, for all $u_{1} \in V_{1}$, the operator $A_{2}\left(u_{1}, \cdot\right): V_{2} \rightarrow 2^{V_{2}^{*}}$ is pseudomonotone on $V_{2}$.

(3) For all $u_{2} \in V_{2}$, there exist an element $w_{1} \in$ $D\left(\widehat{\partial} G_{1}\right) \subset V_{1}$ and a constant $\alpha_{1}>0$ such that

$$
\begin{array}{r}
\left\langle u_{1}^{*}, u_{1}-w_{1}\right\rangle_{V_{1}^{*} \times V_{1}} \geq \alpha_{1}\left\|u_{1}\right\|_{V_{1}}^{2}, \\
\forall u_{1} \in V_{1}, u_{1}^{*} \in A_{1}\left(u_{1}, u_{2}\right) .
\end{array}
$$

(4) For all $u_{1} \in V_{1}$, there exist an element $w_{2} \in$ $D\left(\hat{\partial} G_{2}\right) \subset V_{2}$ and a constant $\alpha_{2}>0$ such that

$$
\begin{array}{r}
\left\langle u_{2}^{*}, u_{2}-w_{2}\right\rangle_{V_{2}^{*} \times V_{2}} \geq \alpha_{2}\left\|u_{2}\right\|_{V_{2}}^{2}, \\
\forall u_{2} \in V_{2}, u_{2}^{*} \in A_{2}\left(u_{1}, u_{2}\right) .
\end{array}
$$

Remark 15. It is clear that the hypotheses (1) and (2) in the assumption (HA) imply that the operator $A$ defined in (21) is also bounded on $V$. The hypotheses (3) on the operator $A_{1}$ and (4) on the operator $A_{2}$ in the assumption (HA) imply the $w_{1}$-coercivity of $A_{1}$ with respect to the first argument and $w_{2}$-coercivity of $A_{2}$ with respect to the second argument, respectively. Moreover, for $w=\left(w_{1}, w_{2}\right) \in D(\widehat{\partial} G)$, the operator $A$ defined in (21) is also $w$-coercive with constant $\beta=\min \left\{\alpha_{1}, \alpha_{2}\right\} / 2$. In fact, for all $\mu \in A(u)$, one has

$$
\begin{aligned}
\langle\mu, u-w\rangle_{V^{*} \times V}= & \left\langle\mu_{1}, u_{1}-w_{1}\right\rangle_{V_{1}^{*} \times V_{1}} \\
& +\left\langle\mu_{2}, u_{2}-w_{2}\right\rangle_{V_{2}^{*} \times V_{2}} \\
\geq & \alpha_{1}\left\|u_{1}\right\|_{V_{1}}^{2}+\alpha_{2}\left\|u_{2}\right\|_{V_{2}}^{2} \\
\geq & \frac{\min \left\{\alpha_{1}, \alpha_{2}\right\}}{2}\|u\|_{V}^{2}, \\
= & \beta\|u\|_{V}^{2},
\end{aligned}
$$

which implies the $w$-coercivity with constant $\beta$ of operator $A$ on $V$.

The assumption $(\mathrm{HJ})$ is as follows.

(1) For all $x_{2} \in X_{2}$, there exist constants $c_{1}, d_{1} \geq 0$ such that

$$
\left\|\eta_{1}\right\|_{X_{1}^{*}} \leq c_{1}+d_{1}\left\|x_{1}\right\|_{X_{1}}, \quad \forall \eta_{1} \in \partial_{1} J\left(x_{1}, x_{2}\right) .
$$

(2) For all $x_{1} \in X_{1}$, there exist constants $c_{2}, d_{2} \geq 0$ such that

$$
\left\|\eta_{2}\right\|_{X_{2}^{*}} \leq c_{2}+d_{2}\left\|x_{2}\right\|_{X_{2}}, \quad \forall \eta_{2} \in \partial_{2} J\left(x_{1}, x_{2}\right) .
$$


Remark 16. It is clear that the hypotheses in assumption (HJ) imply that $\partial_{1} J\left(\cdot, x_{2}\right)$ and $\partial_{2} J\left(x_{1}, \cdot\right)$ are bounded on $X_{1}$ and $X_{2}$, respectively. Moreover, if $J$ is regular on $X$, then $\partial J$ is also bounded on $X$. (In the following, let $X=X_{1} \times X_{2}$ and $X^{*}=$ $X_{1}^{*} \times X_{2}^{*}$ for simplicity of writing.) In fact, since $J$ is regular on $X$, the inclusion $\partial J\left(x_{1}, x_{2}\right) \subseteq \partial_{1} J\left(x_{1}, x_{2}\right) \times \partial_{2} J\left(x_{1}, x_{2}\right)$ holds. It follows from (35) and (36) that

$$
\|\eta\|_{X^{*}} \leq c+d\|x\|_{X}, \quad \forall \eta \in \partial J(x),
$$

with $c=c_{1}+c_{2} \geq 0$ and $d=\max \left\{d_{1}, d_{2}\right\} \geq 0$. This also means that $\partial J$ is bounded on $X$.

We are now in a position to give our main result on the existence of solution to the problem $\left(\mathrm{P}^{\prime}\right)$, a system of two generalized variational-hemivariational inequalities.

Theorem 17. Suppose that the set-valued mappings $A_{i}: V_{1} \times$ $V_{2} \rightarrow 2^{V_{i}^{*}}, i=1,2$, which satisfy the assumption (HA), is such that the operator A defined in (21) is pseudomonotone on $V$. Let $T_{i}: V_{i} \rightarrow X_{i}$ be linear continuous and compact operators, let $J: X \rightarrow R$ be a regular, locally Lipschitz functional which satisfies the hypothesis (HJ), let and $G_{i}: V_{i} \rightarrow R \cup\{+\infty\}, i=$ 1,2 be proper, convex, and lower semicontinuous functionals. Then the problem $\left(\mathrm{P}^{\prime}\right)$ admits at least one solution under the condition

$$
\beta>d\|T\|^{2}
$$

where $\|T\|$ is the norm of the operator $T$ defined by (21).

Proof. By Lemma 13, the existence of solution to the problem $\left(\mathrm{P}^{\prime}\right)$ of a system of two generalized variationalhemivariational inequalities can be proved as long as the problem $\left(\mathrm{P}^{\prime \prime}\right)$ of a generalized vector variationalhemivariational inequality is solvable. Therefore, we consider the following inclusion problem. Find $u \in V$ such that

$$
F(u)+\widehat{\partial} G(u) \ni 0
$$

where $F: V \rightarrow 2^{V^{*}}$ with $F(u)=A(u)+T^{*} \circ \partial J \circ T(u)$ for all $u \in V$. We will prove the existence of solution to the inclusion problem (39) by the surjectivity theorem (Theorem 11), which implies that the problem $\left(\mathrm{P}^{\prime \prime}\right)$ is solvable.

Claim 1 ( $F$ is bounded on $V)$. Since the operator $A$ is bounded on $V$ under assumption (HA) by Remark 15, $\partial J$ is also bounded on $X$ under the assumption $(\mathrm{HJ})$ by Remark 16, and $T$ is linear continuous by the linearity and continuity of the operators $T_{i}, i=1,2$, and it is easy to check that $F$ is bounded on $V$, which implies that $F_{u_{0}}: V \rightarrow 2^{V^{*}}$ with $F_{u_{0}}(u)=F\left(u_{0}+u\right)$ is quasibounded for any $u_{0} \in V$.

Claim 2 ( $F$ is pseudomonotone on $V)$. Since $F=A+T^{*} \circ \partial J \circ T$ : $V \rightarrow 2^{V^{*}}$ and the operator $A$ is pseudomonotone, we only need to prove that $T^{*} \circ \partial J \circ T: V \rightarrow 2^{V^{*}}$ is pseudomonotone. To this end, firstly, we prove that $T^{*} \circ \partial J \circ T$ is generalized pseudomonotone. Let $u^{n} \rightarrow u$ weakly in $V, \xi^{n} \in T^{*}\left(\partial J\left(T u^{n}\right)\right)$ with $\xi^{n} \rightarrow \xi$ weakly in $V^{*}$ and $\lim \sup \left\langle\xi^{n}, u^{n}-u\right\rangle_{V^{*} \times V} \leq 0$.
There exist $\eta^{n} \in \partial J\left(T u^{n}\right)$ such that $\xi^{n}=T^{*} \eta^{n}$. Since $\partial J$ is bounded on $X$ by Remark 16, Tu $\rightarrow T u$ in $X$ by the compactness of the operators $T_{i}, i=1,2$, and $\eta^{n} \in \partial J\left(T u^{n}\right)$, we have the fact that $\eta^{n}$ is bounded in $X^{*}$. Thus there exists a subsequence, which is also denoted by $\eta^{n}$, such that $\eta^{n} \rightarrow \eta$ weakly in $X^{*}$ with some $\eta \in X^{*}$. By using the equality $\xi^{n}=T^{*} \eta^{n}$, it is easy to get that $\xi=T^{*} \eta$. Since $\eta^{n} \in \partial J\left(T u^{n}\right)$ with $\eta^{n} \rightarrow \eta$ weakly in $X^{*}$ and $T u^{n} \rightarrow T u$ in $X$, we get by the closedness of $\partial J$ with $X \times\left(w^{*}-X^{*}\right)$ topology and the reflexivity of $X$ that $\eta \in \partial J(T u)$, and thus $\xi=T^{*} \eta \in$ $T^{*}(\partial J(T u))$. Moreover, it follows from $\eta^{n} \rightarrow \eta$ weakly in $X^{*}$ and $T u^{n} \rightarrow T u$ in $X$ that

$$
\begin{aligned}
\left\langle\xi^{n}, u^{n}\right\rangle_{V^{*} \times V} & =\left\langle\eta^{n}, T u^{n}\right\rangle_{X^{*} \times X} \longrightarrow\langle\eta, T u\rangle_{X^{*} \times X} \\
& =\left\langle T^{*} \eta, u\right\rangle_{V^{*} \times V}=\langle\xi, u\rangle_{V^{*} \times V},
\end{aligned}
$$

which together with $\xi \in T^{*}(\partial J(T u))$ implies that $T^{*} \circ \partial J \circ T$ is generalized pseudomonotone on $V$. Secondly, it is easy to check that $T^{*}(\partial J(T u))$ is nonempty, convex, and closed in $V^{*}$ for all $u \in V$ since $\partial J(x)$ is a nonempty, convex, and closed subset in $X^{*}$ for all $x \in X$ and $T$ is linear and continuous on $V$. Thirdly, the operator $T^{*} \circ \partial J \circ T$ is bounded on $V$, which has been proved in Claim 1. Consequently, it follows from the Proposition 8 that $T^{*} \circ \partial J \circ T$ is pseudomonotone on $V$.

Claim 3 ( $F$ is $w$-coercive on $V$ ). Let $u \in V$ and $\tau \in F(u)$, and then there exist $\mu \in A(u)$ and $\eta \in \partial J(T u)$ such that $\tau=$ $\mu+T^{*} \eta$. By Remarks 15 and 16, we have

$$
\begin{aligned}
\langle\tau, u-w\rangle_{V^{*} \times V} & =\langle\mu, u-w\rangle_{V^{*} \times V}+\left\langle T^{*} \eta, u-w\right\rangle_{V^{*} \times V} \\
\geq & \beta\|u\|_{V}^{2}+\langle\eta, T(u-w)\rangle_{X^{*} \times X} \\
\geq & \beta\|u\|_{V}^{2}-\|\eta\|_{X^{*}}\|T\|\left(\|u\|_{V}+\|w\|_{V}\right) \\
\geq & \beta\|u\|_{V}^{2}-\left(c+d\|T u\|_{X}\right)\|T\|\left(\|u\|_{V}+\|w\|_{V}\right) \\
\geq & \left(\beta-d\|T\|^{2}\right)\|u\|_{V}^{2} \\
& \quad-\|T\|\left(c+d\|T\|\|w\|_{V}\right)\|u\|_{V}-c\|T\|\|w\|_{V}
\end{aligned}
$$

which together with the condition $\beta>d\|T\|^{2}$ means that $F$ is $w$-coercive on $V$ with function $c(t)=\left(\beta-d\|T\|^{2}\right) t-\|T\|(c+$ $\left.d\|T\|\|w\|_{V}\right)-c\|T\|\|w\|_{V} / t$.

It is well known that $\hat{\partial} G$ is a maximal monotone operator on $V$ since, by Lemma 12, the functional $G$ is proper, convex, and lower semicontinuous on $V$ (see [18]). We are now in a position to apply Theorem 11 to the set-valued operators $F$ and $\widehat{\partial} G$. We deduce that $F+\widehat{\partial} G$ is surjective, which implies that there exist $u \in V$ such that

$$
0 \in F(u)+\widehat{\partial} G(u) .
$$

By the definition of the operator $F$, there exist $\mu \in A(u)$, $\eta \in \partial J(T u)$, and $\xi \in \widehat{\partial} G(u)$ such that

$$
\mu+T^{*} \eta+\xi=0
$$


By multiplying the equality (43) by $v-u$ for all $v \in V$, we obtain from the definition of Clarke's generalized subgradient of the functional $J$ and subgradient in the sense of convex analysis of the functional $G$ that

$$
\begin{aligned}
0 & =\langle\mu, v-u\rangle_{V^{*} \times V}+\left\langle T^{*} \eta, v-u\right\rangle_{V^{*} \times V}+\langle\xi, v-u\rangle_{V^{*} \times V} \\
& =\langle\mu, v-u\rangle_{V^{*} \times V}+\langle\eta, T v-T u\rangle_{X^{*} \times X}+\langle\xi, v-u\rangle_{V^{*} \times V} \\
& \leq\langle\mu, v-u\rangle_{V^{*} \times V}+J^{\circ}(T u, T v-T u)+G(v)-G(u),
\end{aligned}
$$

which implies that $u$ solves the problem $\left(\mathrm{P}^{\prime \prime}\right)$ of a generalized vector variational-hemivariational inequality. As stated at the beginning of our proof, $u$ is also a solution to the problem $\left(\mathrm{P}^{\prime}\right)$ of a system of two generalized variationalhemivariational inequalities by Lemma 13 . This completes the proof of Theorem 17.

Remark 18. The pseudomonotonicity of the operator $A$ defined in (21) is necessary for the proof of the existence of solution to the problem $\left(\mathrm{P}^{\prime}\right)$ by the surjectivity theorem since the pseudomonotonicity of operator $A_{1}$ with respect to the first argument and the pseudomonotonicity of operator $A_{2}$ with respect to the second argument, which are necessary to prove the existence of solution to each generalized variational-hemivariational inequality in problem $\left(\mathrm{P}^{\prime}\right)$, cannot guarantee the pseudomonotonicity of the operator $A$ defined in (21) in general. However, some special cases in which the pseudomonotonicity of operator $A_{1}$ with respect to the first argument and the pseudomonotonicity of operator $A_{2}$ with respect to the second argument imply the pseudomonotonicity of the operator $A$ defined in (21) can be given under some stronger conditions (see [5]).

It is obvious that, by similar arguments as proof of Theorem 17, we have the following results for the existence of solution to each generalized variational-hemivariational inequality in the system (3).

Theorem 19. Suppose that, for $i=1,2, A_{i}: V_{1} \times V_{2} \rightarrow$ $2^{V_{i}^{*}}$ are set-valued mappings satisfying the assumption (HA) and that $T_{i}: V_{i} \rightarrow X_{i}$ are linear continuous and compact operators. Let $J: X \rightarrow R$ be a regular, locally Lipschitz functional on $X$, which satisfies the hypothesis (HJ), and let $G_{i}: V_{i} \rightarrow R \cup\{+\infty\}, i=1,2$ be proper, convex, and lower semicontinuous functionals. Then, the ith $i=1,2$ generalized variational-hemivariational inequality in the system (3) admits at least one solution $u_{i} \in V_{i}$ for all $u_{j} \in V_{j}, j \neq i$ under the condition

$$
\alpha_{i}>d_{i}\left\|T_{i}\right\|^{2}
$$

where $\left\|T_{i}\right\|$ is the norm of the operator $T_{i}$.

Remark 20. By comparing Theorems 17 with 19, we remark here that, in addition to the pseudomonotonicity of the operator $A$ defined in (21), we need strongly condition (38) than (45) to obtain the existence of solution to the problem $\left(\mathrm{P}^{\prime}\right)$ of a system of two generalized variationalhemivariational inequalities.
As a natural generalization of Theorem 17 for the existence of solution to the problem $\left(\mathrm{P}^{\prime}\right)$ of a system of two generalized variational-hemivariational inequalities, we can obtain the following theorem for the existence of solution to the problem $(\mathrm{P})$ of a system of generalized variationalhemivariational inequalities concerning set-valued mappings.

Theorem 21. Suppose that the following assumptions on the operators in the problem (P) of a system of generalized variational-hemivariational inequalities hold.

(1) For $i=1,2, \ldots, n, A_{i}: \prod_{k=1}^{n} V_{k} \rightarrow 2^{V_{i}^{*}}$ are set-valued mappings satisfying the following.

(a) $A_{i}$ are bounded on $\prod_{k=1}^{n} V_{k}$ and pseudomonotone with respect to the ith argument.

(b) the operator $A: V=\prod_{k=1}^{n} V_{k} \rightarrow 2^{V^{*}}$, which is defined by $A(u)=\left(A_{1}(u), \ldots, A_{n}(u)\right)$, is pseudomonotone on $V$.

(c) For all $u_{j} \in V_{j}, j \neq i$, there exist an element $w_{i} \in$ $D\left(\widehat{\partial} G_{i}\right) \subset V_{i}$ and a constant $\alpha_{i}>0$ such that

$$
\begin{aligned}
& \left\langle u_{i}^{*}, u_{i}-w_{i}\right\rangle_{V_{i}^{*} \times V_{i}} \geq \alpha_{i}\left\|u_{i}\right\|_{V_{i}}^{2}, \\
& \forall u_{i} \in V_{i}, u_{i}^{*} \in A_{i}\left(u_{1}, \ldots, u_{n}\right) .
\end{aligned}
$$

(2) $J: \prod_{k=1}^{n} X_{k} \rightarrow R$ is a regular and locally Lipschitz functional which satisfies that for all $i=1,2, \ldots, n$ and $x_{j} \in X_{j}, j \neq i$, there exist constants $c_{i}, d_{i} \geq 0$ such that

$$
\begin{gathered}
\left\|\eta_{i}\right\|_{X_{i}^{*}} \leq c_{i}+d_{i}\left\|x_{i}\right\|_{X_{i}}, \\
\forall x_{i} \in X_{i}, \eta_{i} \in \partial_{i} J\left(x_{1}, \ldots, x_{n}\right) .
\end{gathered}
$$

(3) For $i=1,2, \ldots, n, T_{i}: V_{i} \rightarrow X_{i}$ are linear continuous and compact operators and $G_{i}: V_{i} \rightarrow R \cup\{+\infty\}$ are proper, convex, and lower semicontinuous functionals.

Then the problem (P) admits at least one solution under the condition

$$
\widetilde{\beta}>\widetilde{d}\|\widetilde{T}\|^{2}
$$

where $\widetilde{\beta}=\min \left\{\alpha_{i}\right\} / n, \tilde{d}=\max \left\{d_{i}\right\}$ and $\|\widetilde{T}\|$ is the norm of the operator $\widetilde{T}: \prod_{k=1}^{n} V_{k} \rightarrow \prod_{k=1}^{n} X_{k}$ defined by $\widetilde{T}\left(u_{1}, \ldots, u_{n}\right)=$ $\left(T_{1} u_{1}, \ldots, T_{n} u_{n}\right)$.

\section{An Application}

In this section, we are concerned with an application of our results to a system of generalized variational-hemivariational inequalities involving integrals of Clarke's generalized directional derivatives.

Let $\Omega \subset R^{n}$ be a bounded and open set in $R^{n}$, let $V_{1}, V_{2}, \ldots, V_{n}$ be real, separable, and reflexive Banach spaces with dual spaces $V_{1}^{*}, V_{2}^{*}, \ldots, V_{n}^{*}$. For $i=1,2, \ldots, n, A_{i}$ : $\prod_{k=1}^{n} V_{k} \rightarrow 2^{V_{i}^{*}}$ are set-valued mappings, $T_{i}: V_{i} \rightarrow L^{2}(\Omega)$ 
are linear continuous and compact operators on $V_{i}$, and $G_{i}: V_{i} \rightarrow R \cup\{+\infty\}$ are proper, convex, and lower semicontinuous functionals. We consider the following system of generalized variational-hemivariational inequalities involving integrals of Clarke's generalized directional derivatives. For all $i=1,2, \ldots, n$, find $u_{i} \in V_{i}$ and $\mu_{i} \in A_{i}(u)$ such that

$$
\begin{gathered}
\left\langle\mu_{i}, v_{i}-u_{i}\right\rangle_{V_{i}^{*} \times V_{i}}+\int_{\Omega} j_{i}^{\circ}\left(x, \widehat{\mathbf{u}}(x) ; \widehat{v}_{i}(x)-\widehat{u}_{i}(x)\right) d x \\
+G_{i}\left(v_{i}\right)-G_{i}\left(u_{i}\right) \geq 0, \quad \forall v_{i} \in V_{i},
\end{gathered}
$$

where $\mathbf{u}=\left(u_{1}, u_{2}, \ldots, u_{n}\right) \in \prod_{k=1}^{n} V_{k}, \widehat{\mathbf{u}}=\left(\widehat{u}_{1}, \widehat{u}_{2}, \ldots, \widehat{u}_{n}\right)=$ $\left(T_{1} u_{1}, T_{2} u_{2}, \ldots, T_{n} u_{n}\right) \in L^{2}\left(\Omega ; R^{n}\right), \widehat{v}_{i}=T_{i} v_{i}$ and $j(x, y): \Omega \times$ $R^{n} \rightarrow R$ is a function satisfying the following assumption:

$(\mathrm{Hj})$ is as follows.

(1) $j(x, \cdot)$ is locally Lipschitz on $R^{n}$ for a.e. $x \in \Omega$.

(2) $j(x, y): \Omega \times R^{n} \rightarrow R$ is a Carathéodory function.

(3) Either $j(x, \cdot)$ or $-j(x, \cdot)$ is regular on $R^{n}$ for a.e. $x \in \Omega$.

(4) For all $i=1,2, \ldots, n$ and a.e. $x \in \Omega$, there exists constant $c_{i}, d_{i} \geq 0$ such that

$$
|s| \leq c_{i}+d_{i}\left|y_{i}\right|, \quad \forall y \in R^{n}, s \in \partial_{i} j(x, y) \text {. }
$$

Remark 22. The problem (49) we considered in this section includes the problem studied by Panagiotopoulos et al. [19] by using Brouwer's fixed point theorem as a special case where $n=1, A_{i}$ is single-valued and $G_{i}$ is an indicator of a convex subset $K$.

We define a functional $J$ on $L^{2}\left(\Omega, R^{n}\right)$ as follows:

$$
J(u)=\int_{\Omega} j(x, u(x)) d x, \quad \forall u \in L^{2}\left(\Omega, R^{n}\right) .
$$

It follows from Theorem 3.47 in [2] that, under the assumption $(\mathrm{Hj})$ on the function $j, J$ defined by $(51)$ is a locally Lipschitz functional on $L^{2}\left(\Omega, R^{n}\right)$, which satisfies

$$
\begin{aligned}
& J_{i}^{\circ}\left(u, v_{i}\right)=\int_{\Omega} j_{i}^{\circ}\left(x, u(x), v_{i}(x)\right) d x, \\
& \forall u \in L^{2}\left(\Omega, R^{n}\right), v_{i} \in L^{2}(\Omega), \\
& \|\eta\|_{L^{2}(\Omega)} \leq \widetilde{c}_{i}+\widetilde{d}_{i}\left\|u_{i}\right\|_{L^{2}(\Omega)}, \\
& \forall u \in L^{2}\left(\Omega, R^{n}\right), \eta \in \partial_{i} J(u),
\end{aligned}
$$

where $\widetilde{c}_{i}=\sqrt{2} c_{i}|\Omega| \geq 0$ and $\widetilde{d}_{i}=\sqrt{2} d_{i} \geq 0$.

Now, under the conditions (52), we are in a position to apply our result, Theorem 21, to the problem (49), a system of generalized variational-hemivariational inequalities involving integrals of Clarke's generalized directional derivatives. We conclude this section with the following theorem, which gives the existence of solution to the problem (49).
Theorem 23. For the problem (49), a system of generalized variational-hemivariational inequalities involving integrals of Clarke's generalized directional derivatives, one assumes the following.

(1) For $i=1,2, \ldots, n, A_{i}: \prod_{k=1}^{n} V_{k} \rightarrow 2^{V_{i}^{*}}$ are set-valued mappings satisfying the following.

(a) $A_{i}$ are bounded on $\prod_{k=1}^{n} V_{k}$ and pseudomonotone with respect to the ith argument.

(b) the operator $A: V=\prod_{k=1}^{n} V_{k} \rightarrow 2^{V^{*}}$, which is defined by $A(u)=\left(A_{1}(u), \ldots, A_{n}(u)\right)$, is pseudomonotone on $V$.

(c) For all $u_{j} \in V_{j}, j \neq i$, there exist an element $w_{i} \in$ $D\left(\widehat{\partial} G_{i}\right) \subset V_{i}$ and a constant $\alpha_{i}>0$ such that

$$
\begin{aligned}
& \left\langle u_{i}^{*}, u_{i}-w_{i}\right\rangle_{V_{i}^{*} \times V_{i}} \geq \alpha_{i}\left\|u_{i}\right\|_{V_{i}}^{2}, \\
& \forall u_{i} \in V_{i}, u_{i}^{*} \in A_{i}\left(u_{1}, \ldots, u_{n}\right) .
\end{aligned}
$$

(2) $j(x, y): \Omega \times R^{n} \rightarrow R$ is a function satisfying the assumption $(\mathrm{Hj})$.

(3) For $i=1,2, \ldots, n, T_{i}: V_{i} \rightarrow L^{2}(\Omega)$ are linear continuous and compact operators and $G_{i}: V_{i} \rightarrow$ $R \cup\{+\infty\}$ are proper, convex, and lower semicontinuous functionals.

Then the problem (49) admits at least one solution under the condition

$$
\widetilde{\beta}>\widetilde{d}\|\widetilde{T}\|^{2}
$$

where $\tilde{\beta}=\min \left\{\alpha_{i}\right\} / n, \tilde{d}=\sqrt{2} \max \left\{d_{i}\right\}$, and $\|\widetilde{T}\|$ is the norm of the operator $\widetilde{T}: \prod_{k=1}^{n} V_{k} \rightarrow L^{2}\left(\Omega ; R^{n}\right)$ defined by $\widetilde{T}\left(u_{1}, \ldots, u_{n}\right)=\left(T_{1} u_{1}, \ldots, T_{n} u_{n}\right)$.

\section{Acknowledgments}

This work was supposed by the National Natural Science Foundation of China (11101069) and the Open Fund (PLN1104, PLN1102) of State Key Laboratory of Oil and Gas Reservoir Geology and Exploitation (Southwest Petroleum University).

\section{References}

[1] S. Carl, V. K. Le, and D. Motreanu, Nonsmooth Variational Problems and Their Inequalities, Springer, Berlin, Germany, 2007.

[2] S. Migórski, A. Ochal, and M. Sofonea, Nonlinear Inclusions and Hemivariational Inequalities, vol. 26 of Advances in Mechanics and Mathematics, Springer, New York, NY, USA, 2013, Models and analysis of contact problems.

[3] Z. Naniewicz and P. D. Panagiotopoulos, Mathematical Theory of Hemivariational Inequalities and Applications, vol. 188 of Monographs and Textbooks in Pure and Applied Mathematics, Marcel Dekker, New York, NY, USA, 1995. 
[4] P. D. Panagiotopoulos, Hemivariational Inequalities, Springer, Berlin, Germany, 1993, Applications in mechanics and engineering.

[5] Z. Denkowski and S. Migórski, "A system of evolution hemivariational inequalities modeling thermoviscoelastic frictional contact," Nonlinear Analysis. Theory, Methods \& Applications, vol. 60, no. 8, pp. 1415-1441, 2005.

[6] D. Repovš and C. Varga, "A Nash type solution for hemivariational inequality systems," Nonlinear Analysis. Theory, Methods \& Applications, vol. 74, no. 16, pp. 5585-5590, 2011.

[7] S. Carl, "Parameter-dependent variational-hemivariational inequalities and an unstable degenerate elliptic free boundary problem," Nonlinear Analysis, vol. 12, no. 6, pp. 3185-3198, 2011.

[8] S. Carl and D. Motreanu, "Extremal solutions of quasilinear parabolic inclusions with generalized Clarke's gradient," Journal of Differential Equations, vol. 191, no. 1, pp. 206-233, 2003.

[9] Z. Denkowski and S. Migórski, "Hemivariational inequalities in thermoviscoelasticity," Nonlinear Analysis. Theory, Methods \& Applications, vol. 63, pp. 87-97, 2005.

[10] Z. Liu, "Generalized quasi-variational hemi-variational inequalities," Applied Mathematics Letters, vol. 17, no. 6, pp. 741-745, 2004.

[11] Z. Liu and D. Motreanu, "A class of variational-hemivariational inequalities of elliptic type," Nonlinearity, vol. 23, no. 7, pp. 17411752, 2010.

[12] S. Migórski and P. Szafraniec, "A class of dynamicfrictional contact problems governed by a system of hemivariational inequalities in thermoviscoelasticity," Nonlinear Analysis. In press.

[13] P. D. Panagiotopoulos, "Nonconvex energy functions. Hemivariational inequalities and substationarity principles," Acta Mechanica, vol. 48, no. 3-4, pp. 111-130, 1983.

[14] Y. B. Xiao and N. J. Huang, "Generalized quasi-variationallike hemivariational inequalities," Nonlinear Analysis. Theory, Methods \& Applications, vol. 69, no. 2, pp. 637-646, 2008.

[15] Y. B. Xiao and N. J. Huang, "Browder-Tikhonov regularization for a class of evolution second order hemivariational inequalities," Journal of Global Optimization, vol. 45, no. 3, pp. 371-388, 2009.

[16] Y. B. Xiao and N. J. Huang, "Well-posedness for a class of variational-hemivariational inequalities with perturbations," Journal of Optimization Theory and Applications, vol. 151, no. 1, pp. 33-51, 2011.

[17] F. H. Clarke, Optimization and Nonsmooth Analysis, vol. 5, Society for Industrial and Applied Mathematics, Philadelphia, Pa, USA, 2nd edition, 1990.

[18] E. Zeidler, Nonlinear Functional Analysis and Its Applications, Springer, Berlin, Germany, 1990.

[19] P. D. Panagiotopoulos, M. Fundo, and V. Rădulescu, "Existence theorems of Hartman-Stampacchia type for hemivariational inequalities and applications," Journal of Global Optimization, vol. 15, no. 1, pp. 41-54, 1999. 


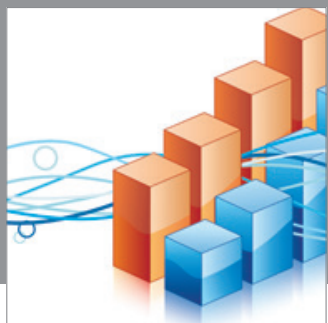

Advances in

Operations Research

mansans

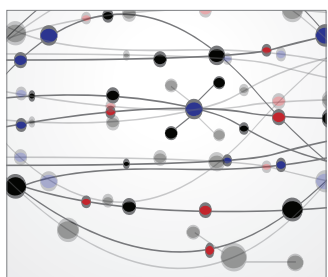

The Scientific World Journal
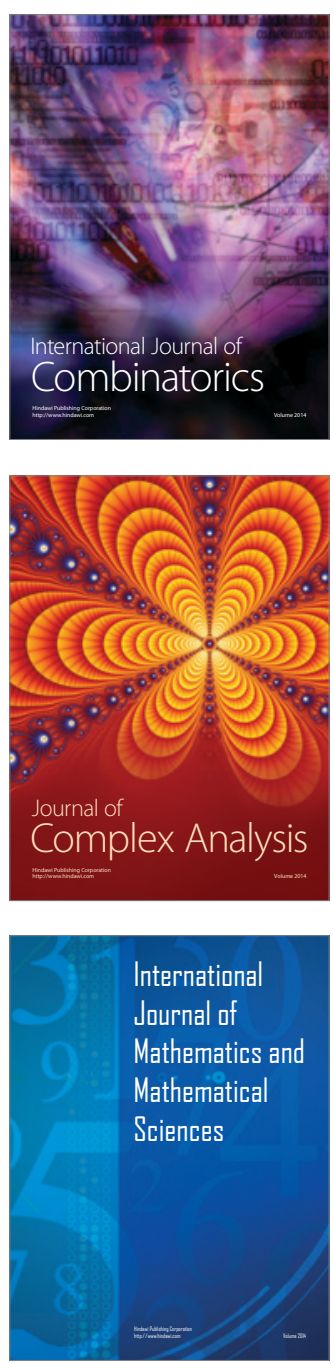
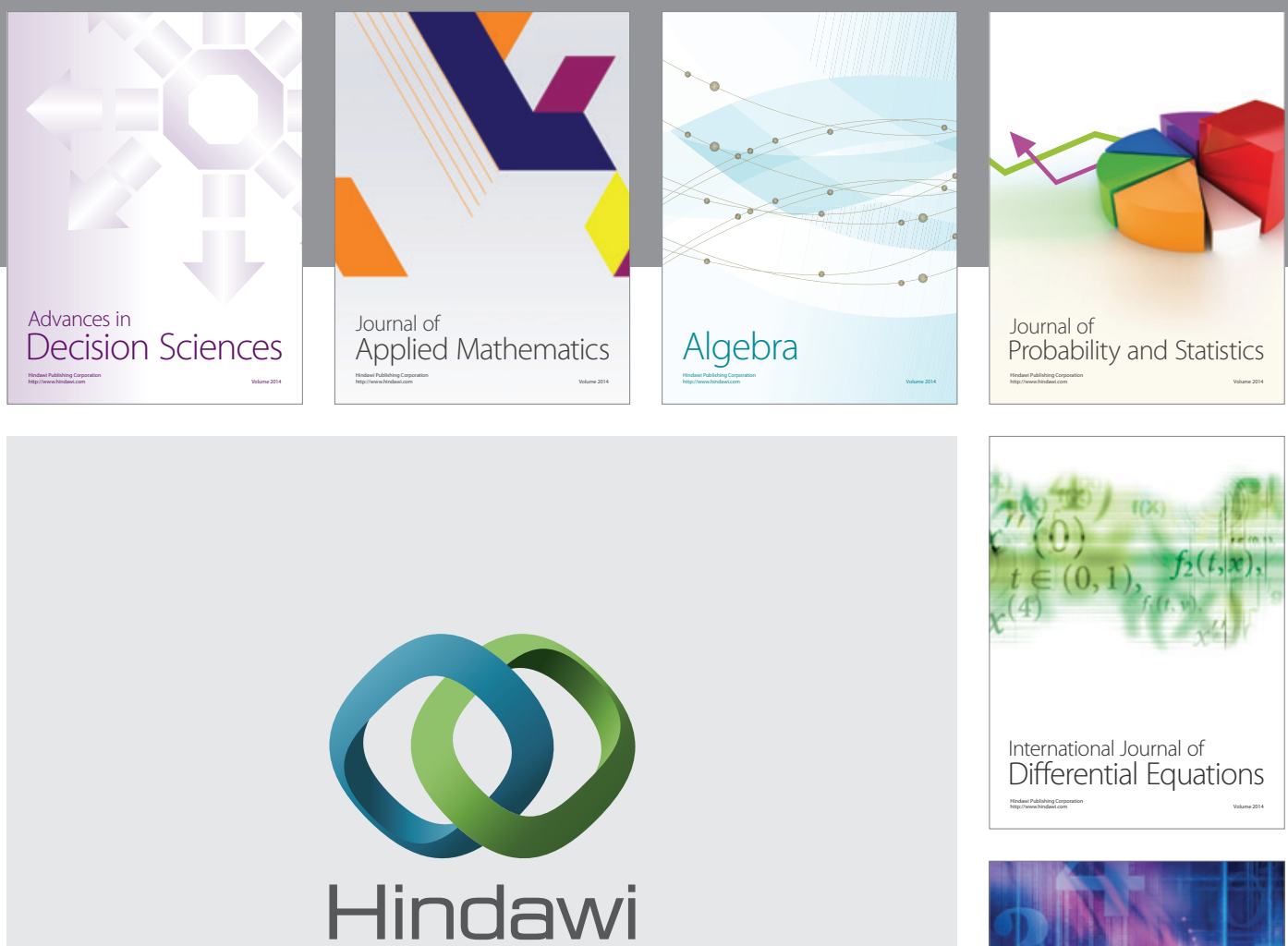

Submit your manuscripts at http://www.hindawi.com
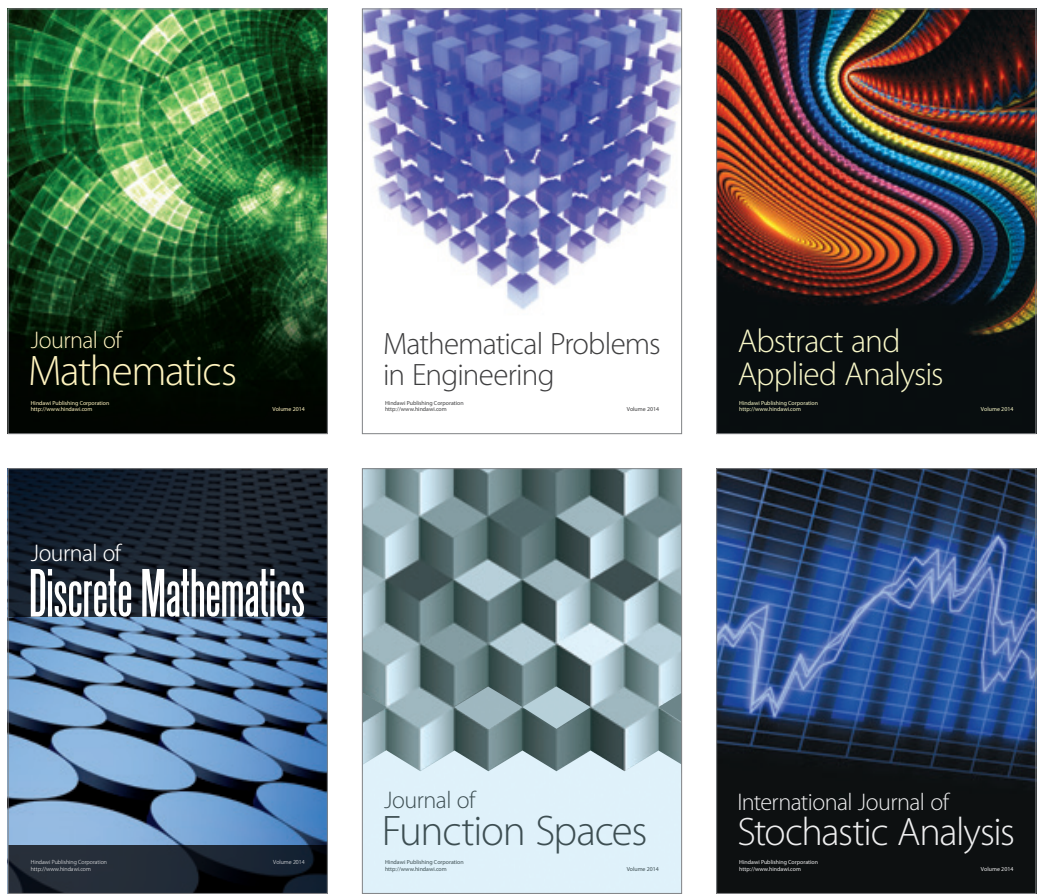

Journal of

Function Spaces

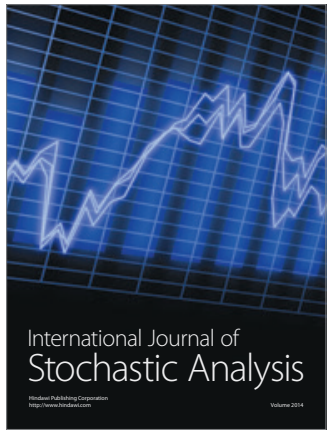

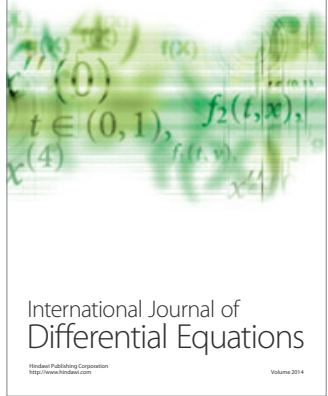
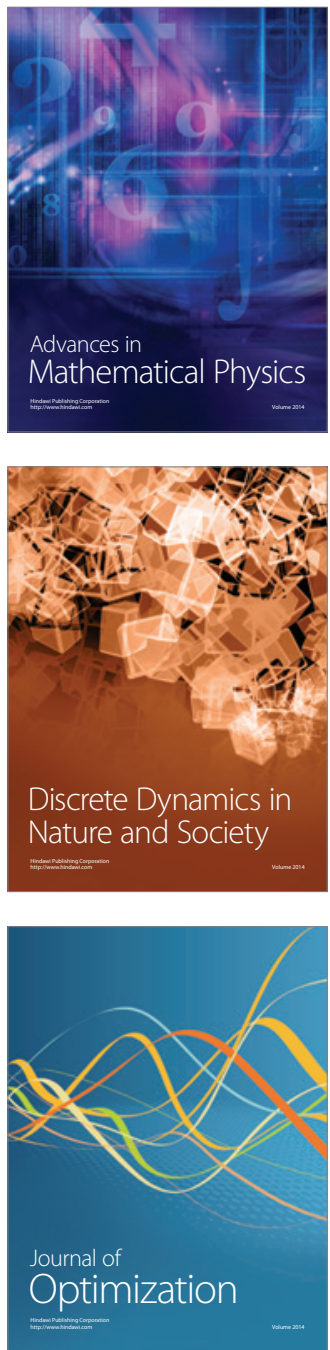